\title{
Parametric oscillation in a vertical microcavity: A polariton condensate or micro-optical parametric oscillation
}

\author{
J. J. Baumberg, ${ }^{1}$ P. G. Savvidis, ${ }^{1}$ R. M. Stevenson, ${ }^{2}$ A. I. Tartakovskii, ${ }^{2}$ M. S. Skolnick, ${ }^{2}$ \\ D. M. Whittaker, ${ }^{3}$ and J. S. Roberts ${ }^{4}$ \\ ${ }^{1}$ Department of Physics \& Astronomy, University of Southampton, SO17 1BJ, United Kingdom \\ ${ }^{2}$ Department of Physics, University of Sheffield, Sheffield S3 7RH, United Kingdom \\ ${ }^{3}$ Toshiba Research Europe Ltd, Cambridge, CB4 4WE, United Kingdom \\ ${ }^{4}$ Department of Electronic and Electrical Engineering, University of Sheffield, Sheffield, S1 3JD, United Kingdom
}

(Received 3 August 2000)

\begin{abstract}
Semiconductor microcavities can support quasiparticles which are half-light and half-matter with interactions possessed by neither component alone. We show that their distorted dispersion relation forms the basis of a quasiparticle "trap" and elicits extreme enhancements of their nonlinear optical properties. When driven by a continuous wave laser at a critical angle, the quasiparticles are sucked into the trap, condensing into a macroscopic quantum state which efficiently emits light. This device is thus an optical parametric oscillator based on quasiparticle engineering. In contrast to a laser, macroscopic coherence is established in the electronic excitations as well as the light field. This paves the way to new techniques analogous to those established in atomic and superconducting condensates, such as ultrasensitive solid-state interferometers.
\end{abstract}

Parametric oscillators are nonlinear resonators in which a coherent pump wave is converted into coherent "signal", and 'idler" waves of different frequency, thus forming the basis for broadband tunable sources and mixers. ${ }^{1}$ They have found widespread application in both microwave and optical frequency regions, as well as providing a "quantum testbed", for some of the most profound demonstrations of nonclassical photon states. ${ }^{2}$ The major stumbling block for optical parametric oscillators (OPO's) has been their inefficient optical nonlinearities, only recently improved with the introduction of periodically patterned media which modify the photon modes. In a similar fashion, the ability to control the wave functions of electrons by tightly confining them inside semiconductor heterostructures has revolutionized the science and technology of light emitters, modulators, and lasers. By combining these approaches a new generation of lightmatter interactions can be built which yield novel science and useful applications. This is most apparent in the vertical cavity surface emitting laser (VCSEL) which uses monolithic integration of a semiconductor quantum well (QW) emitter surrounded by highly reflecting semiconductor Bragg mirror stacks. ${ }^{3}$ By manipulating both the optical and electronic degrees of freedom, low-threshold highly efficient lasing is possible. This planar microcavity design has also shown bistability and amplification. ${ }^{4}$ However, such devices operate in a regime with a large density of excited electronhole pairs whose effect is to broaden the transitions and reduce their coupling to light. In the opposite limit, when highquality microcavities contain sufficient oscillator strength they can enter a new regime due to the strong coupling of light and matter, producing mixed "exciton-polariton", modes split in energy. ${ }^{5,6}$ Much controversy in recent years has centered on whether strongly excited polaritons can show a new type of laser action known as a "boser.,"7-10 This confusion exists because the mixed exciton-photon states appear to possess properties inaccessible to their component particles.
Here we report an optical parametric oscillation of a microcavity (termed a $\mu \mathrm{OPO}$ ) which crucially depends on this exciton-photon physics. Successful shrinking of the shortest previous OPO device by a factor of 10000 results from the formation of a trap for polaritons in the microcavity which efficiently channels energy from the pump. Resonant wave interactions are simultaneously possible for the pump, signal, and idler photons due to the Coulomb interaction between electrons and holes in the semiconductor layers. By looking at light emitted in different directions we directly prove the scattering processes postulated. The threshold power density is to our knowledge lower than any VCSEL emitter. The quantum properties of the microcavity inhibit spontaneous emission into nonoscillating modes, enhance the stimulated gain and produce ultralow threshold operation. The physics in this class of solid-state coherent oscillators is akin to cooperative phenomena such as Bose-Einstein condensation, ferromagnetism, and superfluidity. ${ }^{11}$

Devising an $\mu$ OPO depends crucially on two distinct features: a strong optical parametric interaction, and the efficient photon dynamics of microcavities. Conventionally centimeter-long inorganic crystals are used for nonresonant second-order parametric processes in which a pump photon at $2 \omega$ is split into a signal photon at $\omega+\epsilon$ and idler photon at $\omega-\epsilon$. However current materials are insufficiently nonlinear to allow for submicron interaction lengths. Here another extreme is adopted, using instead a material resonance, in this case the exciton-polariton in a semiconductor heterostructure, for resonant third-order parametric interaction of two pump photons at $\omega$ to produce the signal and idler emission $(\omega \pm \epsilon)$ [Fig. 1(a)]. The free-particle-like dispersion relations of bound electron-hole pairs (excitons) and the dispersion of the confined photon cavity mode are both quadratic [Fig. 1(b)]. Very little scattering can occur between such particles, since energy-momentum conservation is highly restrictive. By strongly coupling together the excitons and photons in a microcavity, two new dispersion branches are produced which have radically different shapes and allow extra scat- 
a
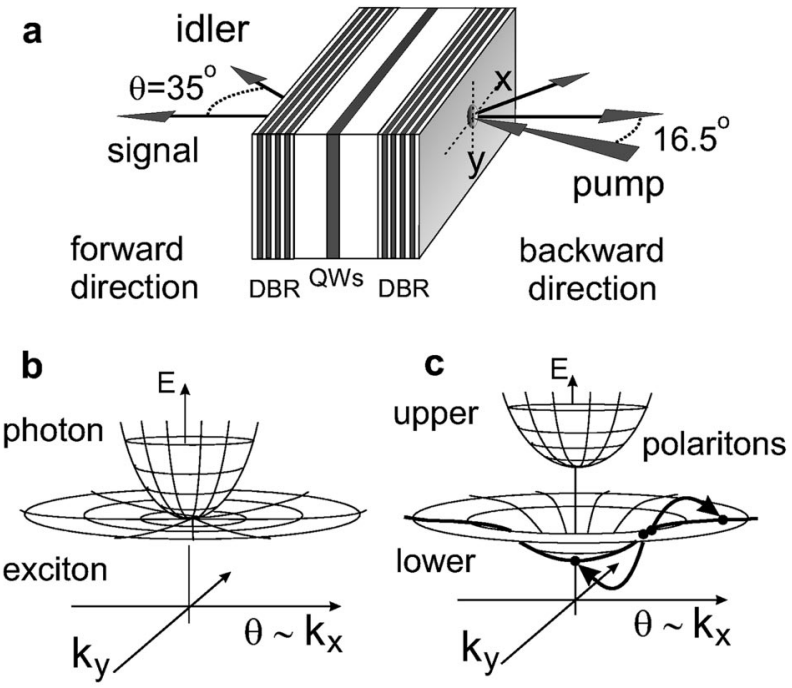

FIG. 1. (a) Geometry of $\mu$ OPO: pump $\left(16.5^{\circ}\right)$, signal $\left(0^{\circ}\right)$ and idler $\left(35^{\circ}\right)$ beams defined by the planar microcavity. Beams emerge in both the forwards and backwards directions with the same inplane momentum. (b) In-plane dispersion relation showing energy of photon and exciton modes vs their in-plane momentum (or incident angle). (c) Modified dispersion of polaritons in the strongcoupling regime. The parametric scattering process shown is only possible for polaritons on the lower branch.

terings to take place, in particular the process shown in Fig. 1(c). Recent pulsed excitation experiments ${ }^{12}$ have transiently revealed the presence of these scattering processes, which can occur when pump polaritons are injected at a critical angle to the microcavity normal, near the turning point of the dispersion. This process has been shown to provide extremely strong gain, building up substantial polariton populations in the lowest states which emit directly out of the sample. A crucial step is the suppression of the normal nonlinear excitonic properties that are inevitably observed at higher excitation densities, in which filling up of the individual electron states prevents their occupation by other electrons, i.e., a fermionic blocking. This is typically seen in the bleaching of the exciton state when strongly exciting a semiconductor. Since lasing requires a large exciton population this would normally prevent any coherence from building up. However, dressing the excitons with photons in a strongly bound "polariton" with a large coherence area, allows them to overlap with bosonic quantum statistics at low densities below those at which the fermionic aspects of electron and hole are manifested. This can be easily identified from the increase of the scattering rate into a polariton state when it is occupied. ${ }^{12}$ The bosonic stimulated scattering of semiconductor polaritons (as opposed to stimulated scattering of photons in a conventional laser) transiently identified in timeresolved measurements leads us to ask whether a polariton condensation process ${ }^{13}$ is possible under $\mathrm{CW}$ excitation.

The semiconductor microcavity [Fig. 1(a)] consists of two pairs of three $10 \mathrm{~nm} \mathrm{In}_{0.06} \mathrm{Ga}_{0.94} \mathrm{As}$ QW's in $10 \mathrm{~nm} \mathrm{GaAs}$ barriers of refractive index $\eta$, sandwiched between 17 (20) pairs of $\mathrm{GaAs} / \mathrm{Al}_{0.18} \mathrm{Ga}_{0.82} \mathrm{As}$ distributed Bragg reflectors (DBRs) on top (bottom). The sample is held in a cold-finger, wide field-of-view cryostat at a temperature of $4 \mathrm{~K}$, allowing access to the dispersion relation of coupled polariton modes
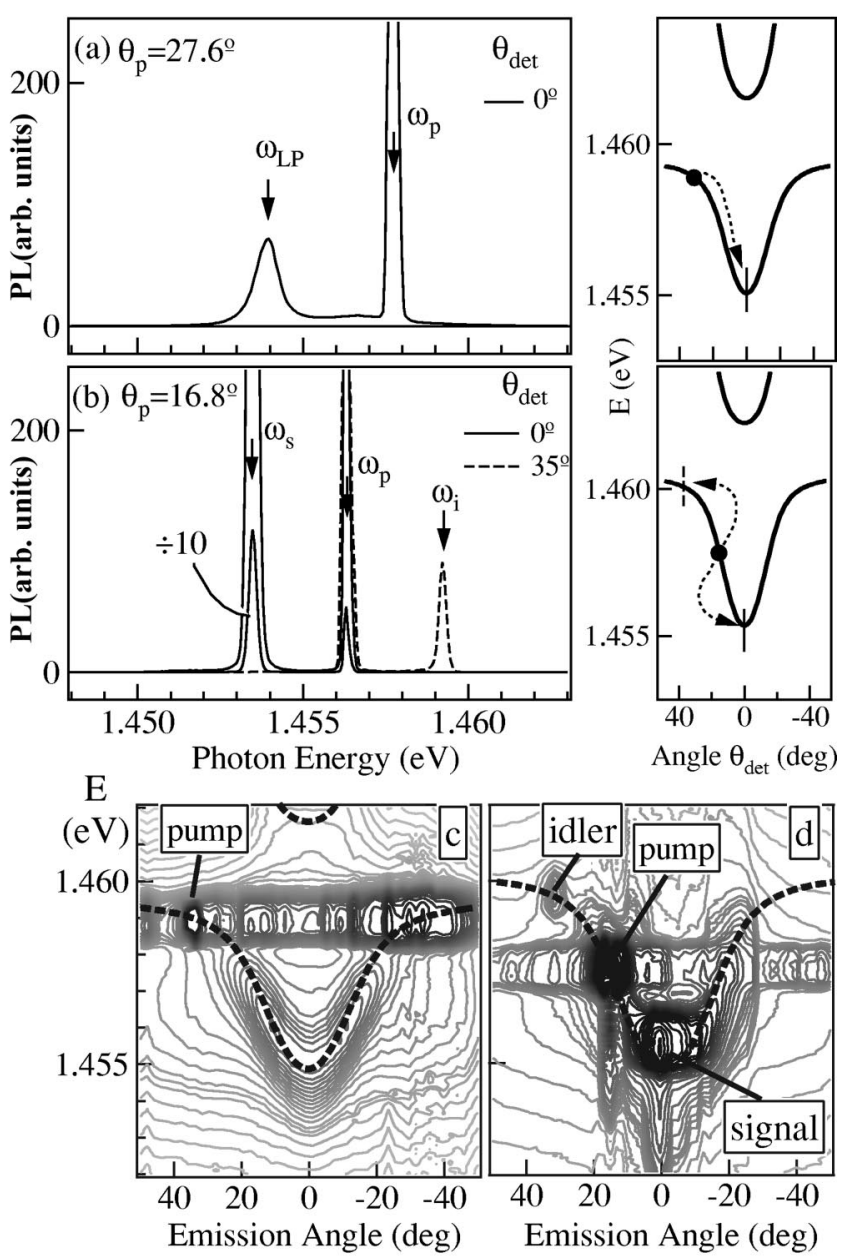

FIG. 2. (a), (b) Photoluminescence spectra at $0^{\circ}$ (signal direction, solid) and $35^{\circ}$ (idler direction, dashed) for a $100 \mathrm{~mW} \mathrm{CW}$ pump incident at (a) $27.6^{\circ}$ and (b) $16.8^{\circ}$ tuned to the lower polariton branch. The elastic pump scatter $\left(\omega_{p}\right)$, lower polariton $\left(\omega_{L P}\right)$, signal $\left(\omega_{s}\right)$ and idler $\left(\omega_{i}\right)$ energies are marked. (c), (d) Contour plots of the emission in energy momentum on a logarithmic scale of eight decades, from a different sample excited at (c) $35^{\circ}$ and (d) $16.5^{\circ}$ with $40 \mathrm{~mW}$, clearly identifying the well-formed polaritons within the $\mu \mathrm{OPO}$ regime. The dispersion curves on the right show the dominant processes.

where different angles of incidence, $\theta$, correspond to different in-plane photon momenta. The effective optical cavity length $\eta d \sim 3 \lambda_{e x} / 2$, where the $\mathrm{QW}$ emission wavelength is $\lambda_{e x}=850 \mathrm{~nm}$, producing a cavity mode at $\lambda_{c}$ $=\lambda_{e x} \sqrt{1-\sin ^{2}(\theta) / \eta^{2}}$ with a finesse $f \sim 1300$. The linewidths of the bare exciton $(0.8 \mathrm{meV})$ and cavity $(1.2 \mathrm{meV})$ modes are much smaller than the polariton splitting of $\hbar \Omega=7 \mathrm{meV}$. Polaritons are injected resonantly at differently angles using a tunable $\mathrm{CW}$ Ti:sapphire laser focused to a spot diameter $\delta=100 \mu \mathrm{m}$, and the central part of the emission is collected in a fiber-coupled goniometer, dispersed in a $0.5 \mathrm{~m}$ monochromator and spectra recorded over 8 decades of intensity using a cooled CCD.

When the lower polariton branch is excited at the exciton energy at large angles $\left(>25^{\circ}\right)$, photoluminescence spectra measured at intervals along the dispersion clearly resolve the two strongly coupled polaritons [Figs. 2(a) and 2(c)]. Light is emitted by the sample within a broad cone concentrated 
within $\pm 20^{\circ}$ due to the effect of the "polariton energy trap" formed by the dispersion relation. This is in complete contrast to the microcavity emission when the pump excites the lower polariton branch at the critical angle $\theta_{c}$ $\simeq \sin ^{-1}\left\{\eta \sqrt{3 \Omega / 2 \omega_{e x}}\right\} \simeq 16.5^{\circ}$. Even at low absorbed pump powers $\left(2 \mathrm{~W} / \mathrm{cm}^{2}\right)$, stronger emission emerges at near normal incidence. At still higher powers $\left(40 \mathrm{~W} / \mathrm{cm}^{2}\right)$, the $\theta=0^{\circ}$ "signal" emission increases rapidly and the $\mu \mathrm{OPO}$ oscillates [Fig. 2(b) and 2(d)]. Also clearly apparent in the spectra is the "idler" mode at $\theta=35^{\circ}$, which is more than an order of magnitude weaker than the signal. Despite the stringent constraints, we observe oscillation at many locations on a sample and show data from two different samples in Fig. 2. The polariton dispersion allows all three polariton modes to be simultaneously resonant in the optical cavity, drastically reducing the threshold of the process by a factor $f_{\text {signal }} f_{\text {pump }} \sim 10^{6}$. Although pairwise polariton creation would imply that equal signal and idler powers are expected, slower photon escape and faster scattering of polaritons at large angles into high momentum states means that far fewer photons are eventually emitted. The spectra in Fig. 2 show that the polariton dispersion is shifted $1 \mathrm{meV}$ to higher energies in the parametric process ${ }^{12,14}$ but the polariton still remains well defined and is not screened out by the large polariton population. This relies on the reduction of the OPO threshold density below the saturation density of excitons. ${ }^{14}$

Key evidence for parametric oscillation is provided by the spectra of this signal beam [Fig. 3(a)], which shows the dramatic superlinear increase of its emission (normalized to the pump power), and the corresponding reduction in linewidth. The spectrally integrated power dependence [Fig. 3(b), filled circles] shows the onset of oscillation, with a threshold which is much less sharp than a conventional OPO, and which has the expected low-power linear behavior of luminescence (dotted), switching to exponential amplification around threshold. The open circles show the almost linear increase of the lower polariton emission when pumping instead the upper polariton. The solid line is a fit to a simple model, ${ }^{15} I_{\text {out }} \propto r-1+\sqrt{(r-1)^{2}+4 r / p}$, which takes into account the number of modes into which polaritons can spontaneously decay $(p)$ and the normalized pump rate, $r$ $=I_{\text {pump }} / I_{t h}$. For optimum conditions, we find the best fit with a threshold pump power absorbed in the microcavity of $1.25 \mathrm{~mW}$ producing an output of $250 \mu \mathrm{W}$ (a $20 \%$ maximum conversion efficiency of absorbed power) with the number of spontaneous modes available, $p \sim 40$. Thus the polariton stimulated parametric scattering forces a significant fraction of the pump polaritons into the normal incidence mode (at the bottom of the trap in the polariton energy dispersion), and hence the need for restricting lateral modes as in a VCSEL no longer exists. In fact, the current planar device has a very similar number of spontaneous modes to high quality laterally defined VCSEL devices. ${ }^{16,17}$

The threshold pump density in the semiconductor heterostructure $\sim 15 \mathrm{~W} / \mathrm{cm}^{2}$ is considerably smaller than that found in the active region of high-quality oxide-apertured VCSEL's (typically several $100 \mathrm{~W} / \mathrm{cm}^{2}$ ), ${ }^{17}$ due to the highly efficient pumping scheme in which the active signal mode "sucks" down carriers from the pump energy through stimulated parametric scattering. We verify this by pumping the microcavity at photon energies above the mirror stop
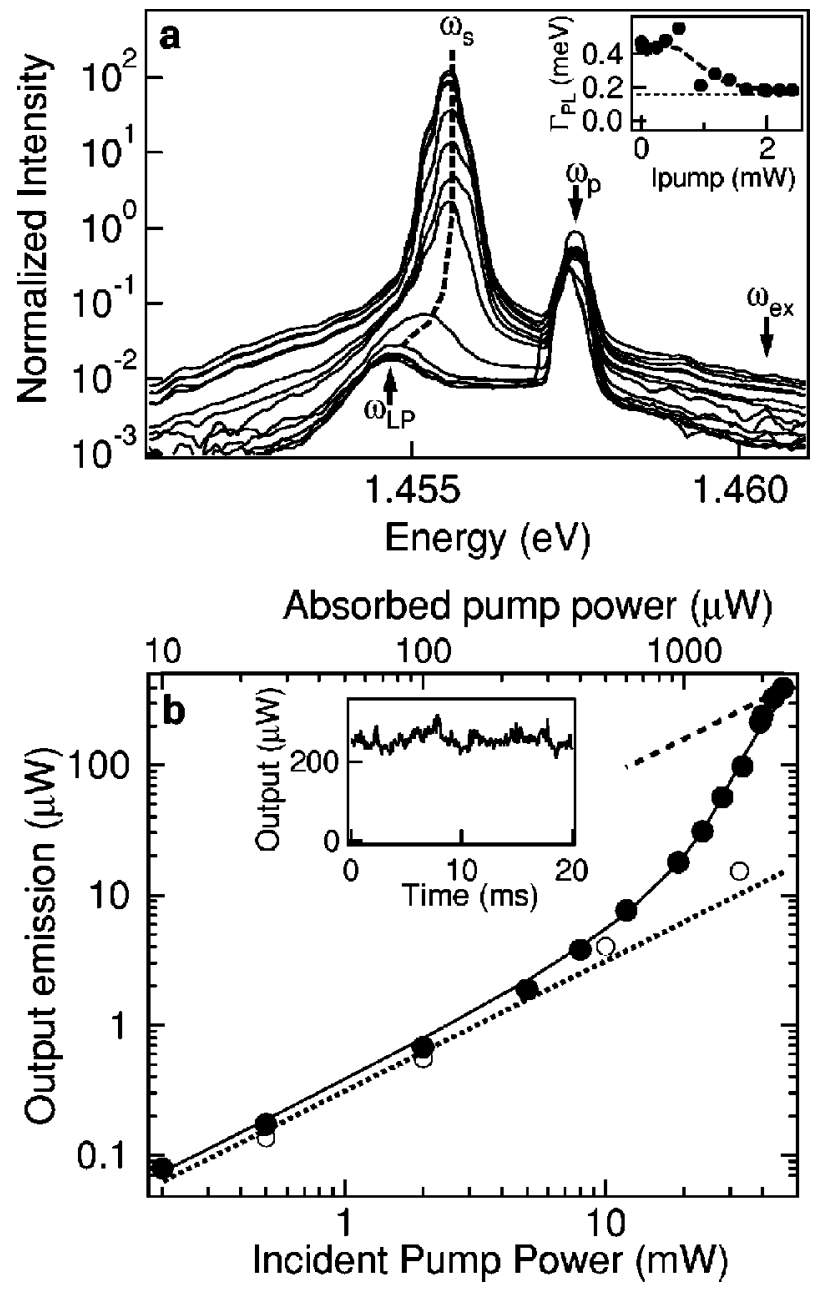

FIG. 3. (a) Power-dependent emission spectra of the signal beam at $0^{\circ}$ normalized to the pump power (incident at $16.5^{\circ}$ ). At high and low powers the emission is linear, while nonlinear at intermediate powers. The energies of lower polariton $\left(\omega_{L P}\right)$, pump $\left(\omega_{p}\right)$, signal $\left(\omega_{s}\right)$ and bare exciton $\left(\omega_{e x}\right)$ are marked. Inset: FWHM emission linewidth vs absorbed pump power. (b) Extracted integrated power output vs incident and absorbed power for excitation at $16.5^{\circ}$ at energies on the lower polariton (solid) and upper polariton (open). The line is a fit described in the text. Inset: Signal beam output.

band so that all the light is absorbed and the microcavity lases as a VCSEL. In this regime, the polariton collapses, ${ }^{8}$ the lasing emission is at the exciton energy, and the threshold is 5 times higher than the $\mu \mathrm{OPO}$ in good agreement with optimized VCSEL's. Parametric oscillation also occurs at other pump angles, though less efficiently, due to multiple parametric scattering. However when resonantly exciting the excitons at large angles, no $\mu \mathrm{OPO}$ behavior is found, which accounts for the failure to observe this mechanism with nonresonant excitation. With a large thermalized population of excitons present, many competing Coulomb-mediated parametric scatterings occur which prevent a sufficient buildup of the lower polariton population. The restricted range of pump angles also shows that in the present case the lasing mechanism is driven by Coulomb scattering between polariton constituents along the dispersion curve, rather than by phonon interactions. Because the coherent emission is clearly at the polariton rather than the exciton energy, the device is not 
simply an exciton laser, but a polariton condensate, with coherence in both exciton and photon components.

The advantage of a microcavity comes from the reduction in the number of cavity modes available for polaritons to emit into, and from the increase in the stimulated scattering rate produced by the resonant mode structure. ${ }^{18-20}$ From measurements of the scattering rates under pulsed excitation conditions, we calculate the threshold incident pump power for the doubly resonant signal and idler $\mu \mathrm{OPO}, I_{t h} \sim 35 \mathrm{~mW}$, in reasonable agreement with our observations. The localization in both energy and angle of the signal beam in dispersion space [Fig. 2(d)] is characteristic for all the conditions we have investigated, with an angular divergence of the signal beam $\Delta \theta \sim 4^{\circ}$ (a fivefold increase over the pump angular width). Microcavity-OPO theory ${ }^{19}$ can account for this angular divergence, which arises from the lateral coherence length of the polaritons in the Airy cavity mode of the FabryPerot cavity, ${ }^{20} l_{c o h}=2 \lambda \sqrt{f p}$, producing an estimated divergence $\Delta \theta_{p}=2 / \sqrt{f p} \approx 3^{\circ}$, in good agreement with our experiment. The lateral coherence length describes the diameter of each polariton, hence macroscopic electronic coherence exists over this length scale. The spontaneous symmetry breaking of the incoherent polariton reservoir when pumped above threshold indicates a well-defined condensate phase whose linewidth is currently not interferometrically resolvable
$(<500 \mathrm{MHz})$. This implies a coherence time $>1 \mathrm{~ns}$, far exceeding the polariton lifetime and unknown for a semiconductor phase coherence. Finally, the quantum statistics of the emitted photons are expected to be highly nonclassical, with a high degree of correlation between the signal and idler photon streams. Future exploitation of the macroscopic electronic coherence is underway to produce high-sensitivity solid-state interferometers.

We conclude that strong coupling between photons and excitons produces judicious quasiparticle dispersions that favor highly efficient $\mu \mathrm{OPO}$ devices containing a macroscopic condensate of polaritons. Similar manipulation of atomic dispersions can also enhance nonlinear interactions. ${ }^{21}$ To further develop room-temperature devices, materials such as $\mathrm{GaN}$ and organic light emitters are promising since they couple more strongly to light and would give larger energy shifts from the pump.

Note added in proof: We have recently observed the polariton angular redistribution accompanying the condensation process. $^{22}$

This work was partly supported by EPSRC GR/M43890, GR/L32187, HEFCE JR98SOBA, and EC CLERMONT HPRN-CT-1999-00132. We would like to acknowledge helpful discussions with C. Ciuti, B. Deveaud, S. Savasta, D. Hanna, and A.C. Tropper.
${ }^{1}$ R. L. Byer, Treatise in Quantum Optoelectronics, edited by H. Rabin and C. L. Tang (Academic Press, New York, 1973).

${ }^{2}$ L. Wu, M. Xiao, and H. J. Kimble, J. Opt. Soc. Am. B 4, 1465 (1987).

${ }^{3}$ T. E. Sale, Vertical Cavity Surface Emitting Lasers (Wiley, New York, 1995).

${ }^{4}$ R. Raj, J. A. Levenson, J. L. Oudar, and M. Bensoussan, Electron. Lett. 29, 167 (1993).

${ }^{5}$ C. Weisbuch, M. Nishioka, A. Ishikawa, and Y. Arakawa, Phys. Rev. Lett. 69, 3314 (1992).

${ }^{6}$ Y. Yamamoto, Nature (London) 405, 629 (2000).

${ }^{7}$ A. Imamoglu et al., Phys. Rev. A 53, 4250 (1996).

${ }^{8}$ M. Kira et al., Phys. Rev. Lett. 79, 5170 (1997); X. Fan, H. Wang, H. Q. Hou, and B. E. Hammons, Phys. Rev. B 56, 15256 (1997)

${ }^{9}$ R. Huang, F. Tassone, and Y. Yamamoto, Phys. Rev. B 61, R7854 (2000); F. Tassone and Y. Yamamoto, ibid. 59, 10830 (1999).

${ }^{10}$ P. Senellart and J. Bloch, Phys. Rev. Lett. 82, 1233 (1999); Le Si Dang et al., ibid. 81, 3920 (1998).

${ }^{11}$ A. J. Leggett, Rev. Mod. Phys. 71, S318 (1999).

${ }^{12}$ P. Savvidis et al., Phys. Rev. Lett. 84, 1547 (2000); P. Savvidis et al., Phys. Rev. B 62, R13278 (2000).

${ }^{13}$ L. V. Keldysh, in Bose-Einstein Condensation, edited by A. Griffin, D. W. Snoke, and S. Stringari (CUP, Cambridge, 1995), p. 246.

${ }^{14}$ C. Ciuti et al., Phys. Rev. B 62, R4825 (2000); C. Ciuti et al., cond-mat/0008408 (unpublished).

${ }^{15}$ A. E. Seigman, Lasers (University Science Books, Sausalito, USA, 1986), pp. 510-522.

${ }^{16}$ F. M. Matinaga et al., Appl. Phys. Lett. 62, 443 (1993).

${ }^{17}$ K. J. Eberling and O. Blum Spahn, in Semiconductor Quantum Optoelectronics, edited by A. Miller, M. Ebrahimzadeh, and D. M. Finlayson (IOP, London, 1999), pp. 309-315.

${ }^{18}$ F. De Martini, F. Cairo, P. Mataloni, and F. Verzegnassi, Phys. Rev. A 46, 4220 (1992).

${ }^{19}$ A. Aiello, D. Fargion, and E. Cianci, Phys. Rev. A 58, 2446 (1998).

${ }^{20}$ G. Bjork, H. Heitmann, and Y. Yamamoto, Phys. Rev. A 47, 4451 (1993).

${ }^{21}$ M. D. Lukin et al., Phys. Rev. Lett. 81, 2675 (1998); M. M. Kash et al., ibid. 82, 5229 (1999).

${ }^{22}$ R. M. Stevenson et al., Phys. Rev. Lett. 85, 3680 (2000). 